\title{
ACOPLAMENTO BIBLIOGRÁFICO NA LITERATURA INTERNACIONAL SOBRE INOVAÇÃO ABERTA NO DESENVOLVIMENTO DE SOFTWARE
}

\author{
Leonardo Pereira Pinheiro de Souza'; \\ Fabiano Armellini ${ }^{2}$
}

\begin{abstract}
Software is ubiquitous in an information-based society. Market complexity requires companies to employ open innovation, incorporating external knowledge and providing knowledge to its' partners. It is aimed to investigate the characteristics of international scientific literature on open innovation and software development, their theoretical affinities and the most relevant authors. As methodological procedures, bibliographic coupling is used, with Social Network Analysis principles, verifying: references in common between papers on this theme, production by country and its annual average. As a result, it is evident that developed countries have higher production on the topic, despite the low annual average. The most referenced papers discuss profitability strategies using open source code. It is concluded that open innovation enables software companies to share knowledge and contribute to the development of society.
\end{abstract}

Keywords: open innovation; software; bibliographic coupling; knowledge; open code.

Resumo: O software é onipresente na sociedade baseada na informação. A complexidade mercadológica requer das empresas a inovação aberta, incorporando saberes externos e cedendo conhecimento aos parceiros. Objetiva-se investigar as características da produção científica internacional sobre inovação aberta e desenvolvimento de software, suas afinidades teóricas e autores mais relevantes. Como procedimentos metodológicos, emprega-se o acoplamento bibliográfico, utilizando princípios da Análise de Redes Sociais, verificando: referências em comum entre trabalhos nessa temática, a produção por país e sua média anual. Como resultados, verifica-se que os países desenvolvidos têm maior produção no tema, apesar da baixa média anual. Os trabalhos mais referenciados discutem estratégias de lucratividade usando código aberto. Conclui-se que a inovação aberta possibilita empresas de software partilharem conhecimentos e contribuírem no desenvolvimento da sociedade.

Palavras-chave: inovação aberta; software; acoplamento bibliográfico; conhecimento; código aberto.

\footnotetext{
${ }^{1}$ Programa de Pós-graduação em Ciência da Informação - Universidade Estadual Paulista (UNESP) Marília - Brasil. E-mail: leopinheirodesouza@gmail.com.

${ }^{2}$ Department of Mathematics and Industrial Engineering - Ecole Polytechnique de Montréal, Montréal - Canadá. Email: fabiano.armellini@polymtl.ca.
} 


\section{INTRODUÇÃO}

Para a sustentabilidade e crescimento econômico é um imperativo investir em tecnologia. O valor dessa tecnologia provém do conhecimento imbuído, referente ao trabalho de Pesquisa e Desenvolvimento (P\&D). A inovação passa a ser imprescindível para a competitividade, em um contexto em que as tecnologias podem ser rapidamente decifradas e replicadas. Contudo, inovar é cada vez mais difícil, em vista da complexidade e rápidas mudanças dos cenários social, mercadológico e científico vigentes (Chesbrough, 2003). Some-se a isto o contexto de globalização, que acirra ainda mais a competição.

Uma estratégia para inovar é unir forças com agentes externos à organização. Esse é o cerne do conceito de inovação aberta (IA), no qual a empresa aproveita o conhecimento disponível externamente, agregando-o ao desenvolvido internamente, inclusive vendendo ou licenciando as próprias descobertas, verificando que novos usos podem ser feitos delas.

A força por trás do desenvolvimento da sociedade contemporânea, embasada na informação e no conhecimento, é a Tecnologia da Informação (TI), destacando o desenvolvimento de softwares. Os softwares estão presentes em praticamente todos os âmbitos da vida cotidiana e algumas das mais lucrativas empresas são baseadas em softwares (Rodriguez, 2020). O software é intangível, constituído principalmente de informação e conhecimento. Como produto tecnológico, a IA torna-se fator fundamental também para o sucesso do software.

São colocados os seguintes problemas de pesquisa: quais os autores mais citados sobre IA no desenvolvimento de software? Quais são as perspectivas preponderantes? Qual o volume dessa produção ao longo do tempo? Como se distribui de acordo com os países de origem?

O objetivo do presente trabalho é investigar as características do corpus de literatura científica internacional concernente à IA no desenvolvimento de software. Por meio de uma análise de acoplamento bibliográfico ( $A B)$, pretende-se verificar quais os autores mais citados nos trabalhos com a temática acima referida, suas perspectivas, e que relações há entre eles. $\mathrm{O} A \mathrm{~B}$ mede a quantidade de referências em comum entre dois ou mais trabalhos, denotando seu nível de proximidade cognitiva (De Bellis, 2009). As referências utilizadas refletem uma mentalidade predominante em um âmbito científico, paradigmas e valores compartilhados. “[...] o registro da 
lista de referências em um trabalho científico reflete o diálogo estabelecido pelo pesquisador com seus pares, bem como o processo de construção do seu conhecimento" (Grácio, 2016, p. 83). Buscase ainda investigar a média de produção de trabalhos ao longo do tempo e como essa produção se distribui entre distintos países.

\section{REFERENCIAL TEÓRICO}

A inovação é essencial para o desenvolvimento econômico das sociedades. A inovação, para Boslaugh (2020), tem papel central no sucesso dos negócios e no progresso da ciência. O investimento em tecnologia agrega alto valor de conhecimento a produtos e serviços, mediante intensas atividades de P\&D. Boslaugh (2020) explana que o economista J. Schumpeter elaborou a teoria da destruição criativa, segundo a qual as inovações propostas pelos empreendedores são a força motriz do crescimento da economia, causando ruptura com as perspectivas anteriores, permitindo a melhoria constante de produtos e serviços. Segundo Fernandes, Ferreira e Peris-Ortiz (2019), durante os primórdios do século XX, imperava o paradigma da inovação fechada, determinando que o controle sobre os recursos intelectuais, produção, comercialização e distribuição, seria a chave para a inovação eficaz, em uma época em que nem os governos nem as universidades estavam envolvidos na aplicação comercial da ciência.

Contudo, as configurações do mundo contemporâneo são bastante distintas. Além das constantes mudanças e tensões sociais, econômicas, políticas e mercadológicas, que trazem imprevisibilidade sobre as configurações da realidade a longo prazo (Bauman, 2001), o mundo está cada vez mais interligado pela globalização e pelos fluxos informacionais via Internet.

É cada vez mais contraproducente tentar inovar somente com recursos internos da empresa. O conhecimento formal, incorporado em produtos e patentes, representa o que Takeuchi e Nonaka (2008) definem como conhecimento explícito, que é codificado, sistematizado e, portanto, mais facilmente compartilhado. Chesbrough (2003) alerta que, pela profusão de conhecimento de fácil acesso, disseminado por meio da Internet, periódicos científicos, instituições de ensino e outros canais, qualquer inovação produzida por uma empresa pode ser, possivelmente, decifrada e reproduzida, perdendo seu diferencial competitivo. A velocidade de perda de exclusividade sobre 
uma inovação é potencializada. Assim, é eficaz criar inovação colaborativamente, renunciando a ilusões de exclusividade vitalícia sobre ela, conforme os princípios da IA.

Para Chesbrough (2003), IA significa a empresa aproveitar o conhecimento produzido no ambiente externo, unindo-o ao conhecimento interno, para obter vantagem competitiva. Contudo, para aproveitar conhecimentos de outras organizações, é preciso criar empatia pessoal e técnica, isto é, entender seus modos de trabalhar e os princípios que regem essas organizações, para melhor adaptar esses saberes à sua própria rotina (Choo, 2003). Ademais, Sivam et. al (2019) afirmam que a IA envolve não apenas relações entre empresas, mas também a colaboração e empoderamento mútuo de funcionários, membros da comunidade e demais stakeholders, ou partes interessadas, visando a superação de desafios e melhoria contínua na organização.

A empresa pode, assim, usar tanto estratégias de internalização quanto de externalização para a IA. Na internalização, o conhecimento flui de fora para dentro. Como recomenda Chesbrough (2003), a organização pode financiar pesquisas acadêmicas ou patrocinar pequenas empresas nascentes, as startups, tirando lições para si dos resultados do trabalho realizado, encontrando novos meios de empregá-los.

Outra possibilidade de inovação é mediante fluxos de conhecimento que partem de dentro para fora da organização. Chesbrough (2003) sugere que a empresa tenha uma postura flexível sobre suas patentes, podendo vendê-las ou licenciá-las, para aprender que novos usos podem ser feitos delas. Até mesmo aquelas descobertas que aparentemente não têm um valor podem se revelar lucrativas (West \& Gallagher, 2006).

É pertinente discutir como as características da empresa se relacionam com a IA. Naqshbandi (2018) elenca os aspectos das empresas bem-sucedidas em IA: conexões com atores internacionais; empresas com mais colaboradores especializados têm maior capacidade de absorver conhecimento e inovar; empresas privadas, particularmente as de propriedade de entidades estrangeiras/internacionais, também inovam mais, pois têm maior acesso a conhecimento e tecnologia de ponta. Para este autor (2018), enquanto que as empresas de grande porte se saem bem em desenvolver a IA tanto via fluxos de internalização de conhecimentos (do ambiente externo para a empresa), quanto via fluxos de externalização (da empresa para o exterior), organizações menores se concentram na internalização, por não terem grande capacidade de P\&D. 
Existem ainda outros fatores críticos para o sucesso da IA. Para Oliveira, Echeveste e Cortimigllia (2018), essas características são: capacidade de liderança, concernente ao gerenciamento eficiente do capital humano; boa gestão dos níveis tático, estratégico e operacional; estrutura e recursos internos para selecionar, priorizar e implementar inovações; capacidade de criar e manter laços formais e informais com agentes e organizações externos; alinhamento entre a estratégia geral da organização e objetivos com sua capacidade inovativa, propiciando a tomada de decisão eficaz; identificar, avaliar e adquirir competências tecnológicas para a inovação, incluindo a gestão de propriedade intelectual; ter uma cultura organizacional que incentive a experimentação, correr riscos, colaboração e partilha de informação.

Entretanto, há atitudes que podem constituir barreiras na absorção de conhecimento externo. Dentre elas: “[...] custos proibitivos de absorção e transação, preocupações com propriedade intelectual, incompatibilidades tecnológicas, ou risco excessivo associado a ideias não comprovadas" (Hannen et al., 2019, pp. 1-2, tradução nossa). Além desses problemas gerenciais, há barreiras psicológicas para os colaboradores utilizarem conhecimento vindo de fora. Segundo Hannen et al. (2019), a 'síndrome do isto não foi inventado aqui', refere-se a uma resistência em usar conhecimentos do meio externo, em que o sujeito rejeita ideias que possam desafiar suas crenças pré-concebidas, ou ainda como fator de ajuste social, quando esse sujeito evita ideias que contrariem a mentalidade prevalecente em seu grupo. Ainda conforme estes autores (2019), essa postura negativa pode ser mitigada por ações de conscientização sobre a importância da IA.

\subsection{ASPECTOS DA INOVAÇÃO ABERTA NO DESENVOLVIMENTO DE SOFTWARE}

É pertinente expor algumas especificidades concernentes ao contexto de desenvolvimento de software. Ressalta-se que, como conclui Souza (2019), profissionais de desenvolvimento de software utilizam intensamente a Internet para obtenção de conhecimentos e troca de experiências com outros profissionais de diversas localidades, por meio dos fóruns de programação. O trabalho colaborativo com agentes externos evidencia-se como um traço marcante nessa cultura profissional. 
Especial destaque pode ser dado às ações de crowdsourcing, definidas como: "a prática de prover ideias, opiniões ou ajuda para desenvolver algo em grande número de pessoas, frequentemente membros do público utilizando a Internet" (Crowdsourcing, 2020, tradução nossa). Pode-se traçar as raízes do fenômeno crowdsourcing em uma tradição mais antiga, que é o desenvolvimento de software open source, ou de código aberto.

Para Raymond (2020), o open source é um movimento social pela descentralização, transparência e compartilhamento de informação, permitindo que códigos-fonte de programas sejam livremente compreendidos e modificados, ao contrário dos softwares comerciais, que não podem ser analisados nem alterados. O open source funciona por um sistema de colaboração aberta:

"[...] um sistema de inovação ou produção que se baseia em participantes orientados para metas, ainda que fracamente coordenados, que interagem para criar um produto (ou serviço) [...] que é feito disponível a colaboradores e não-colaboradores igualmente" (Levine \& Prietula, 2013, p. 2, tradução nossa).

As organizações podem também beneficiar-se de utilizar o crowdsourcing e o open source fazendo parcerias com comunidades de programadores para aumentar seu potencial inovativo. Hill et al. (2017) relatam a aplicação do crowdsourcing e open source para criação de algoritmos mais rápidos para análise de associações de genótipos e fenótipos em estudos do genoma. Segundo estes autores (2017), buscou-se atingir o objetivo com uma maratona em um site de programação, dividindo um prêmio em dinheiro entre os competidores que apresentassem as melhores soluções.

Munir, Runeson e Wnuk (2019) recomendam que empresas de softwares utilizem ferramentas de programação open source, pois deste modo os programadores podem eles mesmos corrigir erros nas ferramentas, sem ter de esperar por atualizações, além de poderem customizá-las conforme suas necessidades. Contudo, estes autores (2019) recomendam que mudanças sejam compartilhadas com a comunidade desenvolvedora da ferramenta, para não gerar problemas de compatibilidade e ainda beneficiar a todos.

Outro fator a ser considerado é a velocidade da inovação. Dong, Wu e Zhang (2019) afirmam que a velocidade em que versões do software, especialmente os open source, são liberadas funciona como um demonstrativo de qualidade para os usuários. Por outro lado, estes autores 
(2019) apontam que softwares que são lançados e/ou atualizados muito rapidamente criam certa incerteza sobre sua consistência. Embora essas conclusões tenham sido feitas sobre software open source, em que é difícil saber como as alterações foram realizadas, já que são feitas por uma multidão anônima, o mesmo efeito psicológico poderia ser esperado com softwares comerciais.

\section{PROCEDIMENTOS METODOLÓGICOS}

A presente pesquisa utiliza o método de $\mathrm{AB}$ para identificar os autores mais utilizados em trabalhos acadêmicos que versam sobre desenvolvimento de software e IA, bem como as relações entre os autores. O princípio fundamental do $\mathrm{AB}$ é que dois artigos estão acoplados se partilham ao menos uma referência em comum, sendo que quanto maior a quantidade de referências compartilhadas maior o grau de acoplamento (De Bellis, 2009).

$\mathrm{O} A B$ permite visualizar a produção científica de um campo do conhecimento por meio das afinidades teórico-metodológicas entre trabalhos. Percebe-se como pensam os pesquisadores, que ideias são mais valorizadas, o que é considerado consenso e os autores mais relevantes, enfim, a estrutura intelectual de um campo do conhecimento (Grácio, 2016).

Em um quadro teórico mais amplo, o AB pode ser compreendido como um método de análise de redes sociais (ARS). Para Otte e Rousseau (2002) a ARS é uma estratégia de investigação de estruturas sociais, onde subjacente a cada rede concreta existe uma estrutura matemática de grafo, formada de nós e arestas, que representam os relacionamentos entre eles. Segundo estes autores (2002), o princípio básico dessa metodologia é o foco nos relacionamentos entre atores (ou nós) da rede, considerando como o comportamento de outros influencia as escolhas individuais. Ademais, de acordo com Silva e Zanata Júnior (2012), a ARS pode usar abordagens tanto qualitativas, como no caso de estudos antropológicos, quanto quantitativas, onde as relações são representadas por valores numéricos, tornando possível sua análise estatística e uso de softwares para seu processamento, como no caso da presente pesquisa.

Para concretização da rede de autores de $\mathrm{AB}$, efetuou-se uma pesquisa na base de dados Web of Science - Clarivate Analytics (WoS), no mês de maio de 2020, utilizando-se os termos: 'open innovation' (inovação aberta); 'programming' (programação) e 'software development' 
(desenvolvimento de software). Como um dos objetivos do presente trabalho é verificar a quantidade de trabalhos ao longo do tempo, não foi estipulado nenhum intervalo de datas para a busca. Os dados de referências dos trabalhos recuperados foram inseridos no software CitNetExplorer para a geração de um gráfico expondo as relações de AB.

O CitNetExplorer tem o objetivo de efetuar análises de redes de citações de publicações individuais, conforme Van Eck e Waltman (2014). Esse software gera um gráfico representativo das relações de citação, no qual os trabalhos são representados por nós interligados por arestas entre os artigos citantes e citados. No plano horizontal, quanto mais próximos estão os nós, maior é a proximidade dos trabalhos, em termos de referências utilizadas em comum. Essa proximidade fica evidenciada com a função de agrupamento (clustering), na qual cada cluster consiste em artigos fortemente conectados entre si por relações de citação, podendo representar um tópico ou tendência na literatura acadêmica (Van Eck \& Waltman, 2014).

Para formar uma visão mais ampla do corpus de literatura considerado, foram também computados: a média de produção por ano; a quantidade de produção por país e os trabalhos mais referenciados, que receberam no mínimo dez citações. Por fim, foram discutidos os pontos principais dos trabalhos mais citados.

\section{RESULTADOS E DISCUSSÃO}

Para desvelar os paradigmas seguidos pelos pesquisadores da IA no desenvolvimento de softwares, foi efetuada uma busca na base WoS, usando os termos: 'open innovation' (inovação aberta); 'software development' (desenvolvimento de software) e 'programming' (programação). Como um dos objetivos do presente trabalho é também verificar a evolução temporal da referida temática, não foi definido nenhum intervalo de tempo para a busca. Como pode ser observado no Quadro 1, foram obtidos cinquenta e cinco resultados, num intervalo de tempo que vai de 2006 a 2020, o que representa uma média de 3,9 trabalhos ao ano. Verifica-se, assim, que apesar de o software ser um elemento fundamental na vida contemporânea, e de a IA ser essencial para competitividade, a produção de trabalhos contemplando esses dois tópicos é tímida. 
Quadro 1- Parâmetros de busca na WoS e resultados.

\begin{tabular}{|l|l|}
\hline \multicolumn{2}{|l|}{ Aspectos da pesquisa } \\
\hline Termos de busca & TS= (“open innovation" AND ("software development" or "programming”)) \\
\hline Campos considerados & Título, resumo e palavras-chave \\
\hline Quantidade de resultados & 55 \\
\hline Anos de publicação & $2006-2020$ \\
\hline Média trabalhos/ano & 3,9 \\
\hline
\end{tabular}

Fonte: elaborado pelos autores (2020).

É pertinente apurar que países têm maior produção na temática considerada, visto que, como já discutido (Seção 2), o investimento em inovação é primordial para o desenvolvimento econômico. Pesquisadores de vinte e cinco países publicaram sobre o tema considerado. Dentre esses países, destacam-se os com maior volume de publicações: Alemanha, com onze trabalhos; Estados Unidos, com nove trabalhos; Suécia com sete trabalhos; Inglaterra com seis trabalhos; Austrália, Canadá e Irlanda com quatro trabalhos. O Brasil não tem nenhum trabalho dentre os resultados recuperados da base de dados. Ressalta-se que o fato de Estados Unidos e Alemanha serem os que mais apresentaram produção na temática considerada possivelmente tem relação com o fato de terem alto investimento em tecnologia e inovação, sendo que a Alemanha destina 3,1\% de seu Produto Interno Bruto a esse fim, e os Estados Unidos destinam 2,8\% (Organisation de Coopération et de Développement Économiques, 2020). Portanto, deve-se considerar que a capacidade inovativa das empresas é afetada pelas políticas nacionais de fomento à inovação. "Esta capacidade incorpora sobretudo o potencial de cada país, nos níveis político e econômico, para produzir fluxos de inovações comercialmente relevantes" (Fernandes, Ferreira \& Peris-Ortiz, 2019, p. 592, tradução nossa). 


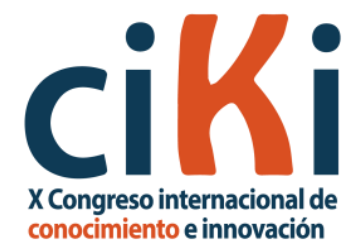

Após a pesquisa na base de dados, foi exportado da WoS um arquivo com dados de referências dos trabalhos, para ser processado pelo CitNetExplorer, gerando o gráfico da Figura 1. Na terminologia da ARS, e da matemática, a figura representa um grafo orientado, no qual cada nó está ligado a outro por uma aresta, que parte do trabalho citado (acima) com destino ao trabalho citante (abaixo) (Otte \& Rousseau, 2002). Aplicando-se a função de clustering da ferramenta, foram formados dois clusters, o um, em azul, e o dois, em verde. O cluster um é o maior, contando com 28 publicações, já o cluster dois contém 15 publicações. Ressalta-se que apenas os quarenta trabalhos mais frequentemente citados são apresentados na visualização, por padrão. Dezoito publicações ficaram desagrupadas (em cinza), visto não estarem fortemente relacionadas por ligações de referências. Pode-se inferir que esses trabalhos representariam autores que não tenham aderido ao consenso prevalecente entre os pesquisadores da temática considerada.

Figura 1 - Rede de citações e acoplamento bibliográfico.

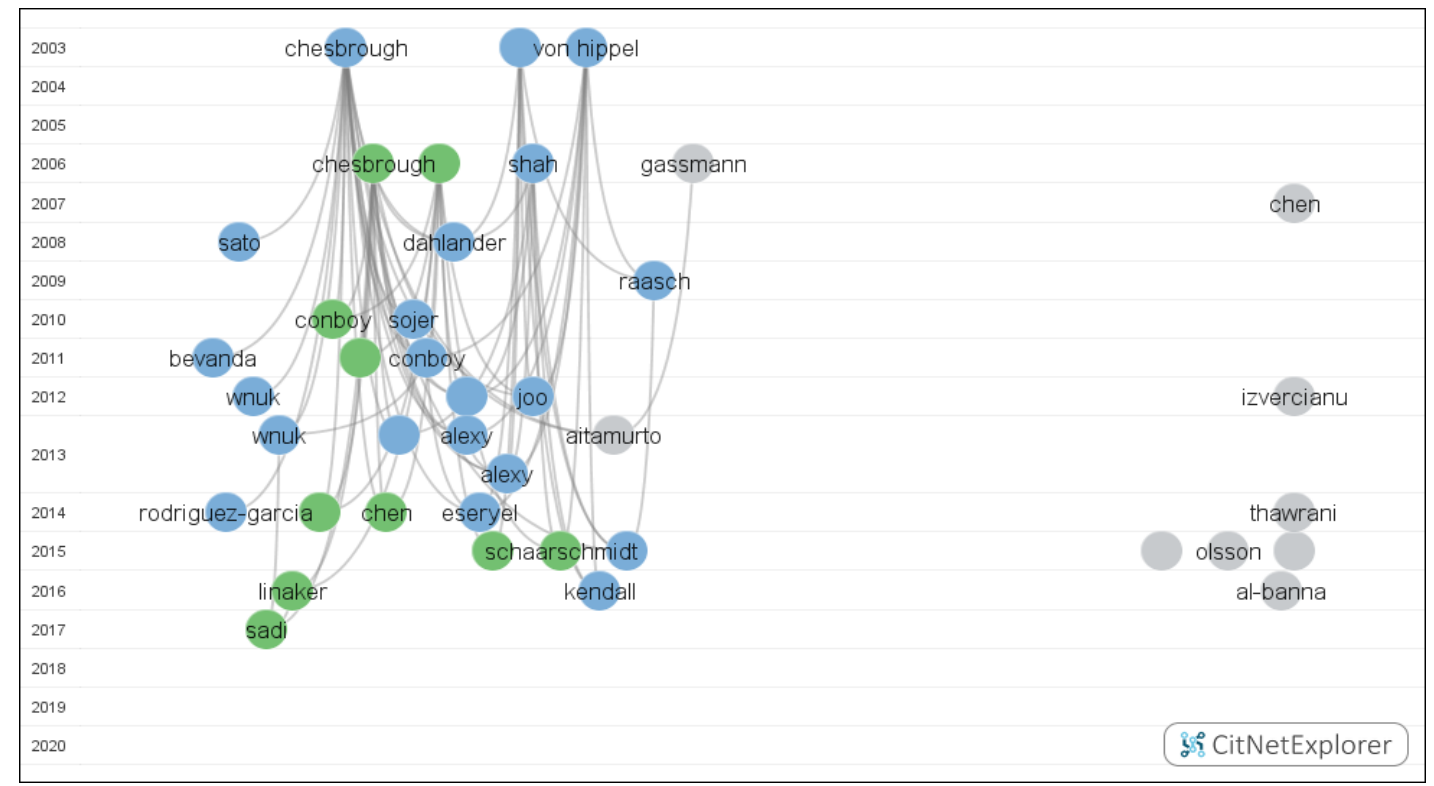

Fonte: elaborado pelos autores (2020).

Para inferir quais seriam os autores mais relevantes em cada cluster, analisou-se o score de citações de cada trabalho, representando "[...] o número de citações de uma publicação dentro da rede de citações que está sendo analisada" (Van Eck \& Waltman, 2014, p. 804). Na ARS, e na teoria dos grafos, essa relevância é ressaltada pelo grau de centralidade, que mede a quantidade de 
arestas adjacentes a um nó (Otte \& Rousseau, 2002). Ressalta-se que ambos os clusters têm Henry Chesbrough como o autor mais citado, sendo esse pesquisador emblemático por seu pioneirismo no estudo da IA. A seguir, o Quadro 2 apresenta a relação dos autores de maior score de citações, ou seja, tendo recebido dez ou mais citações.

Quadro 2 - Scores de citações dos trabalhos mais referenciados.

\begin{tabular}{|l|l|l|}
\hline Autor/trabalho & Score de citações & Cluster \\
\hline Chesbrough (2003) & 22 & 1 \\
\hline Von Hippel \& Von Krogh (2003) & 12 & 1 \\
\hline Lee \& Cole (2003) & 11 & 1 \\
\hline Shah (2006) & 11 & 1 \\
\hline Chesbrough (2006) & 17 & 2 \\
\hline West \& Gallagher (2006) & 14 & 2 \\
\hline
\end{tabular}

Fonte: elaborado pelos autores (2020).

Portanto, segue uma síntese dos pontos principais dos trabalhos mais citados, que denotam os paradigmas partilhados referentes à relação da IA e desenvolvimento de software. Por trazerem uma perspectiva mais ampla sobre a IA, os trabalhos de H. Chesbrough serão comentados ao final, contextualizando os demais.

Ações de IA já ocorriam décadas antes de Henry Chesbrough cunhar esse termo. O contexto em que isso se torna mais evidente é o da chamada 'cultura hacker'. Von Hippel e Von Krogh (2003) afirmam que, desde a década de 1960, programadores costumam produzir e compartilhar programas, dando acesso ao código-fonte, para que sejam analisados e modificados. Estes autores (2003) veem nessa cultura, subjacente ao movimento open source, a base para um modelo privadocoletivo de inovação, em que tanto empresas como a sociedade podem se beneficiar de projetos de código aberto. Um exemplo, já descrito (Subseção 2.1) é o de empresas que contribuem com o desenvolvimento de ferramentas open source para uso próprio, mas liberam as modificações publicamente, para se beneficiarem de aperfeiçoamentos e correções feitos pela comunidade open 
source. Esse modelo de inovação privado-coletivo é uma simbiose, em que se coopera para obter benefícios mútuos, apesar de alguns interesses discrepantes entre as esferas pública e privada.

Lee e Cole (2003) usam o open source para discutir seu modelo de conhecimento baseado em comunidades, que considera a criação do saber como processo em redes, distribuído geográfica e cognitivamente, contrapondo-se ao tradicional modelo de criação de conhecimento, baseado na empresa, debaixo de uma rígida estrutura hierárquica e normativa. Esse é um pensamento afim com o conceito de comunidades de prática, que são formadas espontaneamente por sujeitos de diversas localidades, que se unem para trocar conhecimentos em torno de um assunto de interesse (Wenger, McDermott \& Snyder, 2002). A empresa pode tirar proveito dessas comunidades provendo recursos e tempo para que seus colaboradores participem delas, sem obrigá-los, o que prejudicaria seu ímpeto criativo, segundo Wenger, McDermott e Snyder (2002).

Importante também é apurar as motivações que levam a programadores a dedicarem-se às comunidades open source, para inferir como as empresas podem se acercar dessas essas comunidades e criar parcerias. Shah (2006) identifica que os programadores participam predominantemente pelo prazer do desafio intelectual, além de buscarem benefícios recíprocos da comunidade. Além do modelo open source, Shah (2006) identifica a existência das gated source communities (comunidades de código-fonte fechado), onde membros participam mediante aceite de uma licença corporativa privada, podendo baixar e modificar código, compartilhando-o apenas com usuários licenciados, onde uma empresa toma as decisões de projeto. Ainda na perspectiva desse autor (2006), é importante dar certo grau de liberdade aos programadores, respeitar suas opiniões e não cobrar taxas abusivas, para não tolher seu ímpeto de contribuir.

Por fim, West e Gallagher (2006) classificam a IA em desenvolvimento de software em quatro tipos, destacando o paradigma open source: P\&D compartilhada, no qual empresas doam propriedade intelectual (PI) para projetos open source visando alargar sua capacidade de P\&D e explorar seus benefícios; product development spin-out (giro de desenvolvimento de produto), no qual a empresa abre para a comunidade PIs já sem valor estratégico, para que possam ser transformadas e gerem demanda para seus outros produtos e serviços; venda de complementos, na qual organizações vendem complementos para um produto base que é gratuito; doação de 
complementos, quando a empresa lucra com um produto mas busca colaboração externa para desenvolver complementos que podem ser distribuídos gratuitamente.

É relevante voltar a considerar o trabalho de Chesbrough (2003; 2006), pioneiro em conceituar a IA, elaborando um pensamento coerente, que sintetizou o significado das ações colaborativas para inovação, permitindo às organizações planejarem suas estratégias eficazmente nesse sentido. Para este autor (2003), a empresa que busca aumentar seu arcabouço de conhecimentos, capacitando seu pessoal, efetuando colaborações com benefícios mútuos, tanto para ela como para a sociedade, contribui para o desenvolvimento da comunidade circundante, ajudando a construir um futuro próspero.

A IA, portanto, é um modelo que beneficia não só a empresa, mas a sociedade em que está inserida. Instituições de ensino e pesquisa, governos e a própria classe empreendedora unem esforços para criar e partilhar saberes, gerando novos produtos e serviços que colaboram no desenvolvimento econômico e melhoram, de forma direta ou indireta, a vida das pessoas. Especificamente em relação ao software, destaca-se como a empresa pode ter lucratividade permitindo certa liberdade às comunidades de programadores, para utilizarem, alterarem e distribuírem projetos que atendam às necessidades de um grande contingente de usuários, permitindo o avanço tecnológico.

Ressalta-se que o AB identificou como mais relevantes trabalhos que têm já vários anos de publicados. Isso demostra que as ideias difundidas por esses trabalhos continuam importantes na comunidade de pesquisadores. Esses trabalhos funcionam como um referencial para a evolução do campo, permitindo aos pesquisadores aperfeiçoar, criticar, antagonizar e reinventar os conceitos neles expostos, como demonstram pesquisas mais recentes, já discutidas no presente artigo (Hill et al., 2017; Dong, Wu \& Zhang, 2019; Munir, Runeson \& Wnuk, 2019).

\section{CONSIDERAÇÕES FINAIS}

Discutiu-se a relevância da IA como fator estratégico para a sobrevivência das organizações num contexto globalizado e para o desenvolvimento econômico como um todo. Nesse âmbito, o software tem papel central na sociedade hodierna, que tem na informação e no conhecimento as 
bases de seu desenvolvimento e lucratividade. Portanto, a IA torna-se imprescindível para as empresas que trabalham nesse ramo.

Por meio de uma análise de AB, pôde-se ter um vislumbre das configurações da produção acadêmica sobre a interação entre IA e desenvolvimento de software. Apurou-se que, embora o tema seja social e economicamente relevante, a produção mundial a respeito é ainda tímida e concentra-se em países desenvolvidos, como Estados Unidos e Alemanha. Isto é, possivelmente, reflexo de seu alto investimento do PIB em inovação e tecnologia. Infere-se que esses países mantenham o status de potências justamente por esse investimento.

Constatou-se que a maioria dos trabalhos analisados mantêm estreita afinidade teórica, evidenciada pela partilha de referências em comum. Os autores verificados como mais relevantes, pela quantidade de citações recebidas, convergem em destacar a importância dos projetos open source, executados por meio do crowdsourcing. Enfatiza-se as estratégias utilizadas pelas empresas para conseguirem obter lucratividade e ainda assim apoiarem o livre compartilhamento e alteração de códigos-fonte. Essas ações estão plenamente de acordo com os princípios da IA, que preconizam uma relação de ganhos recíprocos na colaboração entre a empresa e sociedade.

Conclui-se que a IA no desenvolvimento de software tem um papel de enriquecimento mútuo dos arcabouços de conhecimento das empresas e comunidades de programadores, suprindo as necessidades destas, bem como de usuários não-programadores. A IA permite, assim, que empresas de software gerem benefícios diretos na vida das pessoas, quer sejam ou não seus clientes.

\section{AGRADECIMENTOS}

O presente trabalho foi realizado com apoio da Coordenação de Aperfeiçoamento de Pessoal de Nível superior - Brasil (CAPES) - Código de financiamento 001.

\section{REFERÊNCIAS}

Bauman, Z. (2001). Modernidade líquida. Rio de Janeiro: Zahar.

Boslaugh, S. E. (2020). Innovation. In Encyclopadia Britannica. Recuperado de https://academic.eb.com/levels/collegiate/article/innovation/607243. 
Chesbrough, H. (2003). Open Innovation: The New Imperative for Creating and Profiting from Technology. Boston: Harvard Business School.

Chesbrough, H. (2006). Open Business Models: How to Thrive in the New Innovation Landscape. Boston: Harvard Business School Press.

Choo, C. W. (2003). A organização do conhecimento. São Paulo: Editora Senac São Paulo.

Crowdsoursing. (2020). In Macmillan Dictionary. [S.1.]: Macmillan Education. Recuperado de https://www.macmillandictionary.com/dictionary/british/crowdsourcing.

De Bellis, N. (2009). Bibliometrics and citation analysis: From the Science citation index to cybermetrics. Lanham, Md.: Scarecrow Press.

Dong, J. Q., Wu, W. \& Zhang, Y. (2019). The faster the better? Innovation speed and user interest in open source software. Information \& Management, 56 (1), pp. 669-680. Recuperado de https://doi.org/10.1016/j.im.2018.11.002.

Fernandes, C., Ferreira, J. \& Peris-Ortiz, M. (2019). Open innovation: past, present and future trends. Journal of Organizational Change Management, 32 (5), pp. 578-602. Recuperado de https://doi.org/10.1108/JOCM-09-2018-0257.

Grácio, M. C. C. (2016). Acoplamento bibliográfico e análise de cocitação: revisão teóricoconceitual. Encontros Bibli, 21 (47), pp. 82-99. Recuperado de https://doi.org/10.5007/1518-2924.2016v21n47p82.

Hannen, J. et al. (2019). Containing the Not-Invented-Here Syndrome in external knowledge absorption and open innovation: The role of indirect countermeasures. Research Policy, 48 (1). Recuperado de https://doi.org/10.1016/j.respol.2019.103822.

Hill, A. et al. (2017). Stepwise Distributed Open Innovation Contests for Software Development: Acceleration of Genome-Wide Association Analysis. GigaScience, 6 (5), pp. 1-10. Recuperado de https://doi.org/10.1093/gigascience/gix009.

Lee, G. K. \& Cole, R. E. (2003). From a Firm-Based to a Community-Based Model of Knowledge Creation: The Case of the Linux Kernel Development. Organization Science, 14(6), pp. 633-649. Recuperado de: https://doi.org/10.1287/orsc.14.6.633.24866.

Levine, Sheen S. \& Prietula, M. J. (2013). Open Collaboration for Innovation: Principles and Performance. Organization Science. 25 (1), pp. 1414-1433. Recuperado de: https://doi.org/10.1287/orsc.2013.0872.

Munir, H., Runeson, P. \& Wnuk, K. (2019). How Companies Use OSS Tools Ecosystems for Open Innovation. IT Profesional, 21(6), pp. 40-45. Recuperado de https://doi.org/10.1109/MITP.2019.2893134.

Naqshband, M. M. (2018). Organizational Characteristics and Engagement in Open Innovation: Is There a Link? Global Business Review, 19 (3S), pp. 1S-20S. Recuperado de https://doi.org/10.1177/0972150918757845. 
Oliveira, L. S., Echeveste, M. E., Cortimiglia, M. N. (2018). Critical success factors for open innovation implementation. Journal of Organizational Change Management, 31(6), pp. 1283-1294. Recuperado de https://doi.org/1108/JOCM-11-2017-0416.

Organisation de Coopération et de Développement Économiques. (2020). Data: Germany. Recuperado de https://data.oecd.org/germany.htm\#profile-innovationandtechnology.

Otte, E. \& Rousseau, R. (2002). Social network analysis: a powerful strategy, also for the information sciences. Journal of Information Science, 28 (6), pp. 441-453. Recuperado de: https://doi.org/10.1177/016555150202800601.

Raymond, E. S. (2020). Open source. In Encyclopadia Britannica. Recuperado de https://academic.eb.com/levels/collegiate/article/open-source/389944.

Rodriguez, S. (2020, 29 abril). Facebook soars after reporting 'stability' in ad revenue after fall in March, $C N B C$, Tech. Recuperado de https://www.cnbc.com/2020/04/29/facebook-fbearnings-q1-2020.html

Shah, S. K. (2006). Motivation, Governance, and the Viability of Hybrid Forms in Open Source Software Development. Management Science, 52 (7), pp. 1000-1014. Recuperado de https://doi.org/10.1287/mnsc.1060.0553.

Silva, M. K. \& Zanata Júnior, R. (2012). “Diz-me com quem andas, que te direi quem és”: uma breve introdução à análise de redes sociais. Revista USP, 92 (1), pp. 114-130. Recuperado de http://www.revistas.usp.br/revusp/article/view/34893/37629.

Sivam, A. et al. (2019). Key settings for successful Open Innovation Arena. Journal of Computational Design and Engineering, 6 (4), pp. 507-515. Recuperado de https://doi.org/10.1016/j.jcde.2019.03.005.

Souza, L. P. P. (2019). O comportamento informacional dos desenvolvedores de software no contexto da cultura organizacional enfatizando o compartilhamento e reuso de informações (Dissertação de mestrado). Universidade Estadual Paulista, Marília.

Takeuchi, H.; Nonaka, I. (2008). Gestão do conhecimento. Porto Alegre: Bookman.

Van Eck, J. \& Waltman, L. (2014). CitNetExplorer: A new software tool for analyzingand visualizing citation networksNees. Journal of Informetrics, 8 (1), pp. 802-823. Recuperado de http://dx.doi.org/10.1016/j.joi.2014.07.006.

Von Hippel, E. \& Von Krogh, G. (2003). Open Source Software and the "Private-Collective" Innovation Model: Issues for Organization Science. Organization Science, 14(2), pp. 209223. Recuperado de https://doi.org/10.1287/orsc.14.2.209.14992.

Wenger, E., Mcdermott, R. \& Snyder, W. M. (2002). Cultivating communities of practice: A guide to manage knowledge. Boston: Harvard Business School Press.

West, J. \& Gallagher, S. (2006). Challenges of open innovation: the paradox of firm investment in open-source software. $R \& D$ Management, 36 (3), pp. 319-331. Recuperado de https://doi.org/10.1111/j.1467-9310.2006.00436.x. 